\title{
Antimicrobial Properties of Mesenchymal Stem Cells: Therapeutic Potential for Cystic Fibrosis Infection, and Treatment
}

\author{
Morgan T. Sutton, ${ }^{1,2}$ David Fletcher, ${ }^{1}$ Santosh K. Ghosh, ${ }^{3}$ \\ Aaron Weinberg, ${ }^{3}$ Rolf van Heeckeren, ${ }^{1}$ Sukhmani Kaur, ${ }^{1}$ Zhina Sadeghi, ${ }^{4}$ \\ Adonis Hijaz, ${ }^{4}$ Jane Reese, ${ }^{2,5,6}$ Hillard M. Lazarus, ${ }^{2,5,6}$ Donald P. Lennon, ${ }^{2,7,8}$ \\ Arnold I. Caplan, ${ }^{2,7,8}$ and Tracey L. Bonfield ${ }^{1,2}$ \\ ${ }^{1}$ Department of Pediatrics, Case Western Reserve University, Cleveland, OH 44106, USA \\ ${ }^{2}$ National Center of Regenerative Medicine, Case Western Reserve University, Cleveland, OH 44106, USA \\ ${ }^{3}$ School of Dentistry, Case Western Reserve University, Cleveland, OH 44106, USA \\ ${ }^{4}$ Department of Urology, Case Western Reserve University, Cleveland, OH 44106, USA \\ ${ }^{5}$ Department of Cancer Biology, Case Western Reserve University, Cleveland, OH 44106, USA \\ ${ }^{6}$ CTSC Cell Therapy Laboratory, Case Western Reserve University, Cleveland, OH 44106, USA \\ ${ }^{7}$ Department of Biology, Case Western Reserve University, Cleveland, OH 44106, USA \\ ${ }^{8}$ Skeletal Research Center, Case Western Reserve University, Cleveland, OH 44106, USA
}

Correspondence should be addressed to Tracey L. Bonfield; tlb7@case.edu

Received 14 August 2015; Accepted 22 October 2015

Academic Editor: Patricia Pranke

Copyright (C) 2016 Morgan T. Sutton et al. This is an open access article distributed under the Creative Commons Attribution License, which permits unrestricted use, distribution, and reproduction in any medium, provided the original work is properly cited.

\begin{abstract}
Cystic fibrosis (CF) is a genetic disease in which the battle between pulmonary infection and inflammation becomes the major cause of morbidity and mortality. We have previously shown that human MSCs (hMSCs) decrease inflammation and infection in the in vivo murine model of CF. The studies in this paper focus on the specificity of the hMSC antimicrobial effectiveness using Pseudomonas aeruginosa (gram negative bacteria) and Staphylococcus aureus (gram positive bacteria). Our studies show that hMSCs secrete bioactive molecules which are antimicrobial in vitro against Pseudomonas aeruginosa, Staphylococcus aureus, and Streptococcus pneumonia, impacting the rate of bacterial growth and transition into colony forming units regardless of the pathogen. Further, we show that the hMSCs have the capacity to enhance antibiotic sensitivity, improving the capacity to kill bacteria. We present data which suggests that the antimicrobial effectiveness is associated with the capacity to slow bacterial growth and the ability of the hMSCs to secrete the antimicrobial peptide LL-37. Lastly, our studies demonstrate that the tissue origin of the hMSCs (bone marrow or adipose tissue derived), the presence of functional cystic fibrosis transmembrane conductance regulator (CFTR: human, Cftr: mouse) activity, and response to effector cytokines can impact both hMSC phenotype and antimicrobial potency and efficacy. These studies demonstrate, the unique capacity of the hMSCs to manage different pathogens and the significance of their phenotype in both the antimicrobial and antibiotic enhancing activities.
\end{abstract}

\section{Introduction}

Cystic fibrosis $(\mathrm{CF})$ is an inherited, fatal disease in which the cystic fibrosis transmembrane conductance regulator (CFTR: human) gene is mutated, resulting in defective CFTR receptor sodium chloride pump activity. Inefficient CFTR function results in the dysregulated balance of sodium and chloride ions across epithelial cell membranes, increasing mucous viscosity which ultimately contributes to a variety of anomalies including pulmonary infection and gastrointestinal obstruction $[1,2]$. Despite new developments in small molecule correctors and CFTR expression enhancers, 
pulmonary bronchiectasis, bacterial colonization, and the ensuing inflammatory response continue to contribute to lung congestion, pulmonary failure, and death in CF patients $[2,3]$. Human mesenchymal stem cells (hMSCs) secrete bioactive molecules that are anti-inflammatory, antimicrobial, angiogenic, chemotactic, antiapoptotic, and antiscarring $[4,5]$ and have been investigated in several lung disease models including asthma, interstitial pulmonary fibrosis, and adult respiratory distress syndrome $[4,6,7]$. We have previously published the potential therapeutic efficacy of hMSCs in the preclinical murine model of CF lung infection and inflammation [8]. In these studies, wild type (WT) and CFTR deficient mice (CF) were chronically infected with Pseudomonas aeruginosa and followed for several days for clinical score, survival, and weight loss kinetics postMSC treatment. In this in vivo model of CF lung infection and inflammation, hMSCs decreased the bacterial burden and thereby enhanced the ability of the CF lung to resolve the infection potentially through changes in the in vivo production of the antimicrobial peptide LL-37. These results were similar to the studies done with hMSCs in sepsis [9].

Although Pseudomonas aeruginosa is the most prevalent bacteria in CF infections and the one most studied in the context of $\mathrm{CF}$, it is not the only bacteria colonizing $\mathrm{CF}$ patients. Other organisms such as Streptococcus pneumoniae and Staphylococcus aureus also create a complex pulmonary niche and microbiome for the CF lung $[10,11]$. Our studies were designed to begin to understand the antimicrobial activity of the hMSCs and the mechanisms associated with the antimicrobial specificity of the hMSCs and their products. We show that the antimicrobial potency of the hMSCs can impact the outcome of infections associated with not only Pseudomonas aeruginosa but also Staphylococcus aureus and Streptococcus pneumoniae. Further, the hMSCs enhance antibiotic potency against each of these pathogens by slowing pathogen growth rate and the production of the antimicrobial peptide LL-37. Our studies explore the impact of CFTR activity on hMSC action, the tissue source of hMSCs, and whether the hMSCs can be optimized for antimicrobial potency and efficacy. In summary, our studies show that hMSC antimicrobial potential depends on their phenotype, which can be optimized for complex multicomponent therapeutic applications.

\section{Methods}

2.1. Cell Preparations. This study was approved by the Case Western Reserve University and University Hospitals of Cleveland Institutional Review Board (IRB) and Ethics Committees. Human posterior iliac crest bone marrow samples were obtained after written informed consent from paid volunteers under IRB, \#CASE12Z05. Human MSCs (hMSCs) were expanded in ex vivo culture per previously published methods $[12,13]$. Human adipose derived MSCs (hADSC) were isolated from elective abdominal liposuction discarded tissue of obese $(25<\mathrm{BMI}<30)$, otherwise healthy, volunteers also under an institutionally approved IRB (\#UH 03-11-22). The dispersed adipose tissue was rinsed with phosphate buffered saline (PBS) and was then incubated in a solution containing $0.1 \%$ collagenase type IA (AldrichSigma, St. Louis, MO) for 1 hour at $37^{\circ} \mathrm{C}$ while being shaken vigorously. The digested tissue was centrifuged at $6000 \mathrm{~g}$ for $10 \mathrm{~min}$ at room temperature. The pellet at the bottom of tube (stromal vascular fraction, SVF) was then suspended in Dulbecco's-Modified Eagle's Medium (DMEM) supplemented with $10 \%$ fetal bovine serum (FBS, nonselected, Biomed Corporation, Foster City, CA) and $10 \mathrm{~mL}$ of F-12 nutritional media (DMEM : F-12, 1:1) and plated at a density of 1000 cells $/ \mathrm{mm}^{2}$. The culture dish was placed in a $5 \%$ $\mathrm{CO}_{2}$ incubator for 6-8 days to allow for the formation of MSC colonies, which were then trypsinized and propagated. MSCs were validated for the ability to produce chondrocytes and by flow cytometry as previously described [14]. hMSC supernatants: hMSCs were grown to confluence and put into serum-free, antibiotic-free conditions for 3 days prior to harvesting the conditioned medium from the confluent cells. Controls of medium alone were used in all of the experiments. All hMSC preparations were utilized at either cell passage 2 or passage 3 and were validated for the ability to produce chondrocytes and phenotyped by flow cytometry as described previously [15]. We did not test the adipose and osteodifferentiation of the hMSCs since our studies specifically evaluated the predifferentiation potential of the hMSCs to be bioactive antimicrobial contributors prior to differentiation. The chondrocyte protocols are used to define the healthy and potential functionality of the preparation.

2.2. Bacteria. Pseudomonas aeruginosa (PA M5715) is a clinical strain obtained with consent [16]. Staphylococcus aureus (ATCC \#25923) and Streptococcus pneumoniae (ATCC \#49619) were grown in Lysogeny Broth (LB), using each of the pathogens in log phase of growth defined by their growth curves $[15,17,18]$. Bacteria were also evaluated for viability and growth profiles prior to utilization in the studies. Bacteria were grown in a BSCL-2 facility which was approved by the Case Western Reserve University Department of Laboratory Safety.

2.3. Animal Studies. All animal studies were done with institutional approval (Case Western Reserve University IACUC: 2014-0093) with each study being done at least 4 times. Mice were used between the ages of 8 weeks to 12 weeks without distinction between sexes. All mice were housed in our CF Lung Infection and Inflammatory Core procedure room prior to and during the entire course of the experiments. Animals were housed in groups of 5 mice/cage, with husbandry maintained by both the Core and the Case Western Reserve Animal Resource Center. Cystic fibrosis transmembrane conductance regulator deficient animals (Cftrtikth $, C f t r, n=$ 10/study) and congenic background controls (C57BL/6J, WT, $n=10$ /study) were anesthetized with IACUC approved ketamine analgesic to minimize discomfort and to maintain pulmonary physiology. Agarose beads impregnated with the pathogens were inoculated into the lungs of the mice using transtracheal administration. All animals received $10^{5}$ Pseudomonas aeruginosa, or Staphylococcus aureus as defined by postculture of the agarose beads. 24 hours after infection, 
mice were infused with $10^{6}$ adult bone marrow derived hMSCs through the retroorbital sinus. We have found that this route allows for direct deposition into the lung [8]. Animals were followed with daily weights and clinical scores out to 10 days, with an assessment within 2 hours of the time of the initial infection. Mice were housed in our separate animal procedure room monitored by the Core Staff and the Animal Resource Center according to the Case Western Reserve University guidelines. The lung bacteria load was evaluated by bronchoalveolar lavage (BAL) for BAL fluid and whole tissue lung homogenates for Pseudomonas aeruginosa or Staphylococcus aureus colony forming units (CFUs) streaking both tissue sources onto Tryptic Soy Agarose (TSA) plates $(20 \mathrm{~mL}$ agar/plate) over a variety of dilutions cultured for 24 hours. Each of the models were used to investigate how the hMSCs affected the response of the murine CF model to gram negative (Pseudomonas aeruginosa) and gram positive (Staphylococcus aureus) organisms, respectively. Animals were identified by numbers to minimize bias between experimental groups. We did not do in vivo studies with Streptococcus pneumonia, although a different pathogen is also a gram positive organism and would be a redundant use of animals.

2.4. Bacteriology. Bacteria were combined with $2 \mathrm{~mL}$ of hMSC supernatants derived from several unidentified donors $(n \geq 4)$ with or without the presence of the antibiotics geneticin, tobramycin, or ceftazadine $(100 \mu \mathrm{g} / \mathrm{mL}$ antibiotic concentrations), using individual cultures for each donor. After incubation, the bacteria were serially diluted in phosphate buffered saline (PBS) to dilutions of $10^{-6}-10^{-9}$ followed by growth on Tryptic Soy Agar plates $(10 \mu \mathrm{L} /$ column, for Pseudomonas aeruginosa and Staphylococcus aureus). MacConkey plates were utilized for Streptococcus pneumoniae. Bacteria CFUs were counted after 24 hours for the time-point specimens. For all of the studies, $1 \mathrm{~mL}$ aliquots of the different bacterial combinations were evaluated for the number of live and growing bacteria measured by ATP luminescence with Bac-Titer Glo assays (Madison, WI) using an ATP $(\mathrm{pg} / \mathrm{mL})$ standard curve and quantified using 5-parameter statistics. $1 \mu \mathrm{M}$ ATP was prepared in culture medium. 10fold serial dilutions in Tryptic Soy Broth (TSB) were used for standard curve. $100 \mu \mathrm{L}$ of sample was added to luminometer plate with $100 \mu \mathrm{L}$ of Glo substrate with analysis using Nikon Luminometer (luciferase, 10-second analysis, no injection).

2.5. Secreted Antimicrobial Peptides. Supernatants from cultured hMSCs were evaluated for LL-37, human beta defensins (hBD) hBD-2, and hBD-3 using ELISA methodology. The levels of LL-37 were determined utilizing a commercially available kit (Hycult, Biotech, Plymouth Meeting PA), following manufacturing instructions. The ELISA assays for human beta defensin (hBD) type 2 and 3 (hBD-2, hBD-3, resp.) were done following previously published methods $[19,20]$. Data is expressed as mean ( $\mathrm{pg} / \mathrm{mL} \pm \mathrm{SEM}, n \geq 4)$.

2.6. Data Evaluation and Statistics. Data is expressed as mean \pm standard error of the mean (SEM) through nonparametric
Mann-Whitney tests. Analysis of variances and linear correlations were performed using GraphPad Prism 6 (La Jolla, CA). Percent error was calculated through use of Student's $t$-tests or analysis of variance as indicated. All significant values were defined as the value of $P \leq 0.05$. In some cases $P$ values which are close to the $5 \%$ confidence interval are given to demonstrate trends towards significance. Analyses of log or square-root transformation were used to compare experimental conditions at a single point with paired $t$ tests and slopes over time, using different stem cell donors as replicates. In the animal infection models, the bacteria counts were compared using stratified log-rank tests, with the stem cell donors being correlated with the outcomes of the in vivo resolution of bacteria load posttreatment. When comparing antimicrobial LL-37 production or Cftr gene expression levels, we used paired $t$-tests, assuming standard deviations of 0.75 on the $\log _{2}$ scale as suggested by Simon $[21,22]$. A 2.8-fold difference in expression can be detected with $80 \%$ power with a two-sided $t$-test at the 0.01 significance level. When the data could not be transformed to normality, a nonparametric van-Elteren test (van Elteren, 1960) $[23,24]$ was used to compare groups, stratifying on the donors. All statistical analyses were done with the assistance of the Statistical Resource Center, Department of Pediatrics.

\section{Results}

3.1. In Vivo Antimicrobial Potency and Efficacy. C57BL/6J and Cftr ${ }^{\text {tmIKth }}$ knockout mice were infected with either Pseudomonas aeruginosa or Staphylococcus aureus followed 24 hours later with an infusion of $10^{6}$ bone marrow derived hMSCs through the retroorbital sinus. The mice were then monitored for 10 days prior to determining their infection status. Any animals that died during the study were documented and held for bacteriology by necropsy. BAL from the Cftr ${ }^{\text {tmlKth }}$ mice (CF) had significantly higher CFUs (in log scale) than the C57BL/6J (WT) regardless of whether infections were associated with Pseudomonas aeruginosa (Figure 1(a); $P \leq 0.05$ for each, $n=10$ ) or Staphylococcus aureus (Figure 1(b); $P \leq 0.05$ for each, $n=10$ ). In either of the two infection models, the CFUs were significantly decreased with hMSC therapy ( $P \leq 0.05$ for each, $n=4$ different hMSC preparations).

3.2. MSCs Potency and Efficacy on Bacterial Growth. Bone marrow derived hMSC supernatants were evaluated for their ability to alter Pseudomonas aeruginosa (Figure 2), Staphylococcus aureus (Figure 3), and Streptococcus pneumoniae (Figure 4) growth rate and survival in vitro using geneticin antibiotics $(100 \mu \mathrm{g} / \mathrm{mL})$ as a positive control.

3.2.1. Pseudomonas aeruginosa (Figure 2). In evaluating the impact of bone marrow derived hMSC secreted soluble factors on Pseudomonas aeruginosa growth (Figure 2(a)), we found that hMSC supernatants decreased Pseudomonas aeruginosa numbers from $41 \pm 3 \mathrm{CFUs}$ (mean \pm SEM, $n=4$ different hMSC donor preparations) to $21 \pm 2$ CFUs $(P<0.05)$. Geneticin decreased Pseudomonas aeruginosa 


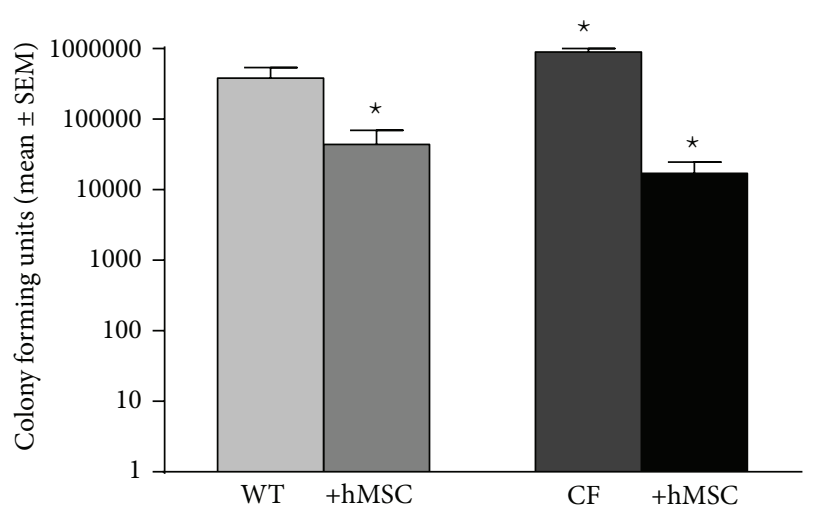

(a) BAL Pseudomonas aeruginosa CFUs

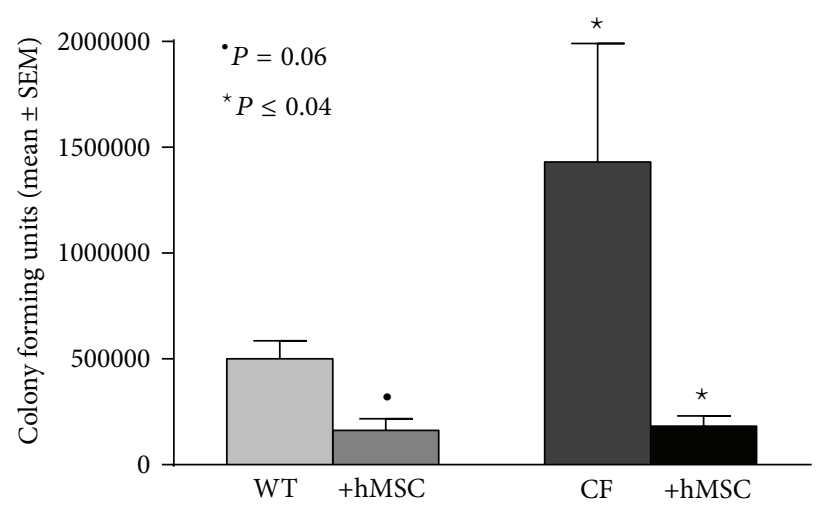

(b) BAL Staphylococcus aureus CFUs

FIGURE 1: hMSCs in the PA (a) and SA (b) infection model: Cftr ${ }^{\text {tmlKth }}$ (CF) and wild type (WT) controls were infected with $10^{5}$ CFUs of either Pseudomonas aeruginosa or Staphylococcus aureus impregnated into agarose beads to generate chronic gram negative or gram positive chronic infection models in CF. hMSCs were administered on day 1, 24 hours after infection. Mice were followed up to 10 days and were then euthanized for bacteria burden (BAL CFUs+ whole lung homogenate CFUs, $n=4$ experiments with 10 animals in each group). hMSCs decreased bacteria burden $(P \leq 0.05)$ in response to both pathogens.

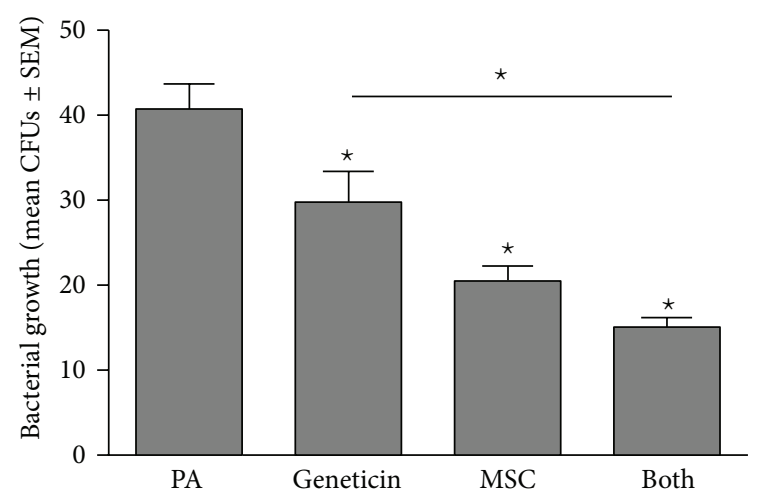

(a) Pseudomonas aeruginosa CFUs

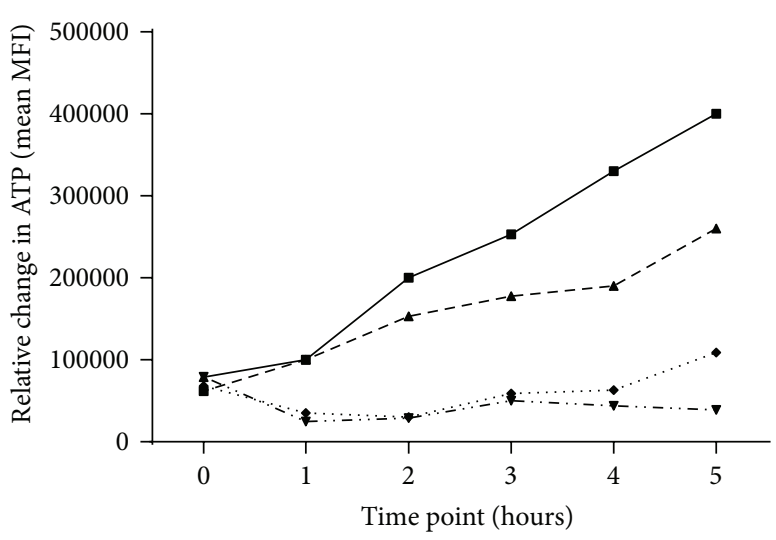

$\rightarrow$ PA and condition medium

- - PA and antibiotics

-.. PA and MSC supernatant

*. PA and MSC supernatant with antibiotics

(b) Pseudomonas aeruginosa growth rate

FiguRE 2: MSCs products decrease Pseudomonas aeruginosa growth. Bone marrow derived hMSCs supernatants were cultured with different dosages of Pseudomonas aeruginosa with and without the addition of geneticin $(100 \mu \mathrm{g} / \mathrm{mL})$. Aliquots of the bacteria were streaked onto TSA plates for CFUs (a) or evaluated for ATP production (b). hMSC supernatants $(n=8$ different donors) significantly decreased both Pseudomonas aeruginosa growth kinetics $(P \leq 0.05)$ and CFUs $(P \leq 0.05)$. Geneticin was used as a positive control which also significantly decreased both CFUs $(P \leq 0.05)$ and growth rate $(P \leq 0.05)$ which was enhanced by the addition of hMSCs $(P \leq 0.05$ versus antibiotic alone for both CFUs and growth kinetics). $\mathrm{PA}=$ Pseudomonas aeruginosa growth without treatment. Geneticin = treatment with the antibiotic geneticin, +hMSCs = treatment with hMSC derived supernatant, and Both = treatment with both hMSC supernatants and geneticin.

growth to $30 \pm 4$ CFUs, which, when combined with hMSC supernatants, decreased Pseudomonas aeruginosa growth to $15 \pm 1(P<0.05)$. To determine the impact of the hMSC supernatants on the growth rate of Pseudomonas aeruginosa, we measured bacterial ATP production (mean MFI \pm SEM, $n=4$ ) versus time (up to 24 hours) focusing on the slope and duration of the hMSC effectiveness. Pseudomonas aeruginosa without hMSC supernatants had a slope of $36 \pm$ 6 (Figure 2(b)). Treatment of the Pseudomonas aeruginosa with the geneticin slowed the growth rate of the bacteria to a slope of $26 \pm 6$, while hMSC supernatant treatment of the pathogen decreased the slope to $-51 \pm 10$ which was significantly less when compared to the bacterial growth rate without treatment $(P<0.05, n=4)$. When combining the geneticin and MSC supernatants there was an additive effect, decreasing the Pseudomonas aeruginosa growth rate to a slope of $-68 \pm 11$, statistically less than either hMSC or geneticin alone $(P<0.05$ for each, $n=4)$. 


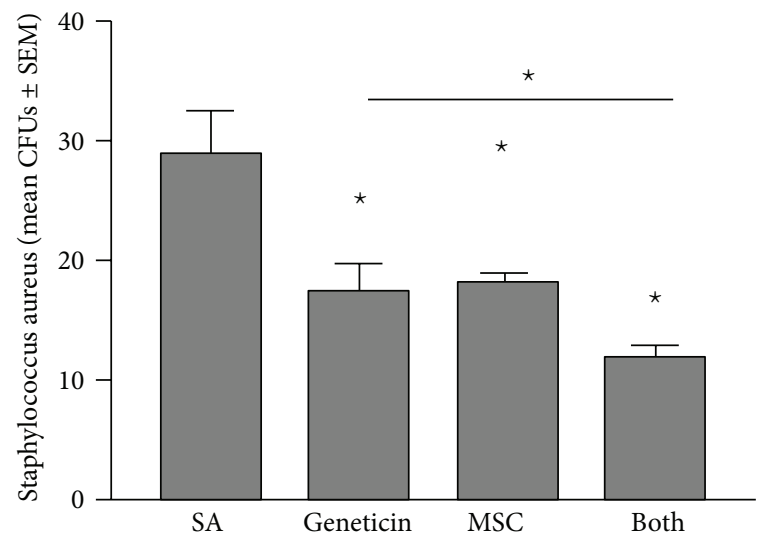

(a) Staphylococcus aureus CFUs

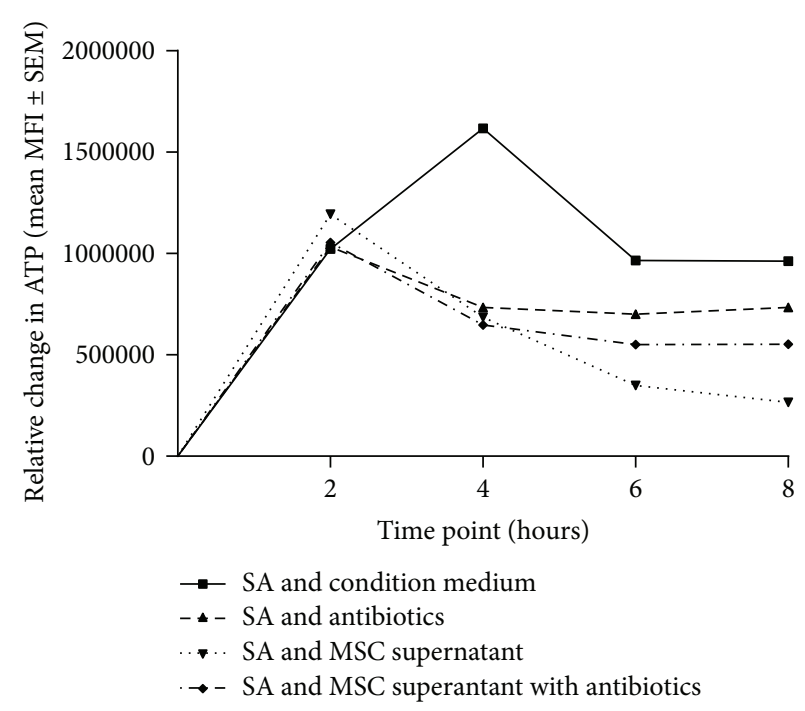

(b) Staphylococcus aureus growth rate

FIGURE 3: MSCs and their products alter Staphylococcus aureus growth. Bone marrow derived hMSCs supernatants were cultured with different dosages of Staphylococcus aureus with and without the addition of geneticin $(100 \mu \mathrm{g} / \mathrm{mL})$. Aliquots of the bacteria were streaked onto TSA plates for CFUs (a) or evaluated for ATP production (b). hMSC supernatants ( $n=8$ different donors) significantly decreased both Staphylococcus aureus CFUs $(P \leq 0.05)$ and growth kinetics $(P \leq 0.05)$. Geneticin was used as a positive control which also significantly decreases both CFUs $(P \leq 0.05)$ and growth rate $(P \leq 0.05)$ which was enhanced by the addition of hMSCs $(P \leq 0.05$ versus antibiotic alone for both CFUs and growth rate). SA = Staphylococcus aureus growth without treatment. Geneticin = treatment with the antibiotic geneticin, + hMSCs $=$ treatment with hMSC derived supernatant, and Both $=$ treatment with both hMSC supernatants and geneticin.

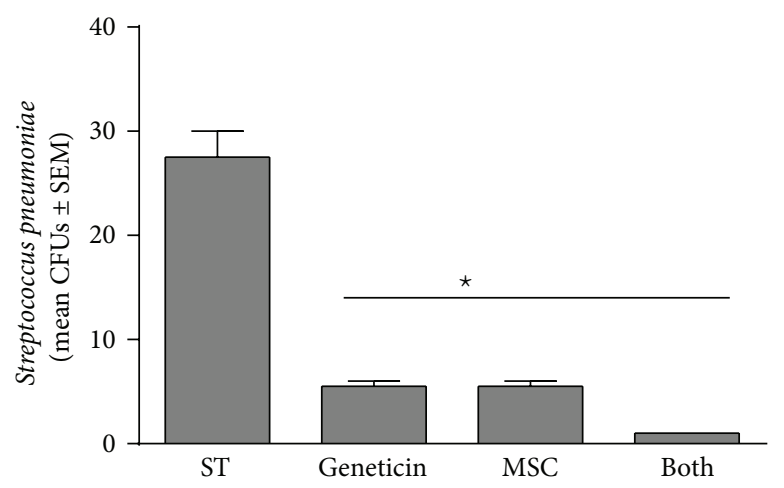

(a) Streptococcus pneumoniae CFUs

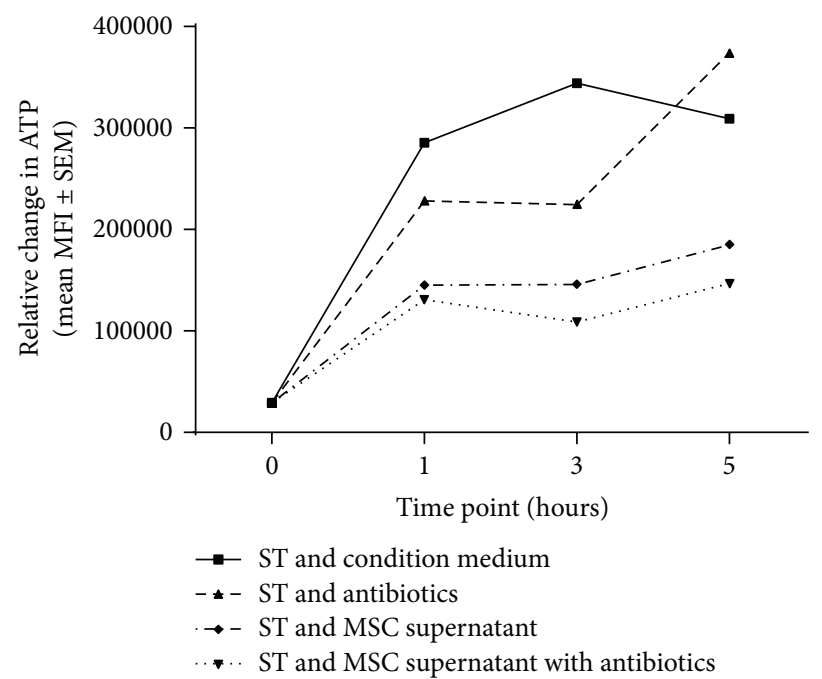

(b) Streptococcus pneumoniae growth rate

FIGURE 4: hMSCs and their products on Streptococcus pneumoniae growth. Bone marrow derived hMSCs supernatants were cultured with different dosages of Streptococcus pneumoniae with and without the addition of geneticin $(100 \mu \mathrm{g} / \mathrm{mL})$. Aliquots of the bacteria were streaked onto MacConkey plates for CFUs (a) or evaluated for ATP production (b). hMSC supernatants $(n=8$ different donors) significantly decreased both Streptococcus pneumoniae CFUs ( (a), $P \leq 0.05)$ and growth rates $((\mathrm{b}), P \leq 0.05)$. Geneticin was used as a positive control, decreasing both CFUs $(P \leq 0.05)$ and growth rates $(P \leq 0.05)$. hMSC supernatants decreased CFUs and enhanced antibiotic sensitivity when measuring CFUs. However, hMSCs supernatants had minimal antibiotic enhancing effect on Streptococcus pneumonia growth rate (b). ST = Streptococcus pneumoniae growth without treatment. Geneticin = treatment with the antibiotic geneticin, $+\mathrm{hMSCs}=$ treatment with hMSC derived supernatant, and Both $=$ treatment with both MSC supernatants and geneticin. 
3.2.2. Staphylococcus aureus (Figure 3). The ability of the bone marrow derived hMSCs to impact the CFUs of Staphylococcus aureus was consistent with the Pseudomonas aeruginosa CFU data. Staphylococcus aureus at baseline resulted in $27 \pm 7$ CFUs (Figure 3(a)), whereas treatment of the bacteria with geneticin or hMSC supernatants had $15 \pm 5$ CFUs or 18 \pm 2 CFUs, respectively $(P<0.05, n=4$ hMSC donor supernatants). When the hMSC supernatants were combined with the geneticin, the impact on Staphylococcus aureus growth was additive, resulting in $12 \pm 2$ CFUs, which was statistically less than either hMSCs supernatants treatment or antibiotic alone ( $P \leq 0.05$ for each, $n=4$ hMSC supernatants).

The impact of the hMSC on Staphylococcus aureus growth rate was different than Pseudomonas aeruginosa (Figure 3(b)). It took almost 4 hours before a noticeable change in Staphylococcus aureus growth was observed, even when comparing each of the conditions of medium alone, geneticin, hMSC supernatant, and when hMSC supernatant and geneticin were combined. The hMSC supernatants or antibiotic impact on the bacterial growth reached significance at 6 hours and was sustained until 8 hours. During this window of hMSC treatment, the number of viable Staphylococcus aureus was significantly decreased with the geneticin $(P<0.05, n=4)$ or hMSC supernatant $(P<0.05)$ which when combined together was additive in antimicrobial effectiveness $(P<$ $0.05, n=4$ comparing to baseline and each of the conditions alone). This data is consistent with the observation that MSCs are antimicrobial against both Pseudomonas aeruginosa and Staphylococcus aureus in scenarios of wound healing $[9,25$, 26].

3.2.3. Streptococcus pneumoniae (Figure 4). Similar to the Staphylococcus aureus and the Pseudomonas aeruginosa studies, the bone marrow derived hMSC supernatants decreased Streptococcus pneumoniae CFUs $(P \leq 0.05)$ as did the geneticin (Figure 4(a), $P \leq 0.05$ ). In addition, like the two other pathogens, there was an enhancing effect of the combination of the hMSC supernatant with the antibiotic on decreasing Streptococcus pneumoniae growth $(P \leq$ 0.05). When evaluating the impact of hMSC supernatants on Streptococcus pneumoniae survival and growth rate, the response of the pathogen was unique when compared to both the Pseudomonas aeruginosa and the Staphylococcus aureus (using the same hMSC derived supernatants). The impact of the hMSC supernatants on the Streptococcus pneumoniae was not apparent until 1 hour and reached significance at 3 hours (Figure 4(b), $P<0.05, n=4$ different hMSC supernatants). Further, by 5 hours the geneticin appeared to have become ineffective, while the hMSC supernatants sustained efficacy (duration of the antimicrobial effect). Although the geneticin $(P<0.05, n=4)$ and the hMSC supernatant $(P<0.05$, $n=4)$ had comparable antimicrobial effectiveness against Streptococcus pneumoniae, there was an additive effect (hMSC supernatant and geneticin) on Streptococcus pneumoniae ATP production starting at 1 hour, which was consistent with Figure 4(a). However the additive effect of hMSC supernatant and geneticin was short-lived, lasting only until 2 hours posttreatment. There was no statistically sustained benefit of combining the hMSC supernatant and antibiotic on growth rate, suggesting that the hMSC antimicrobial impact on Streptococcus pneumoniae growth rate was through different mechanisms than the hMSC supernatant effect on the other two pathogens, especially since the studies were done using the same hMSC supernatant donors, as well as antibiotics. The reasons for the differences are a focus of ongoing studies in the laboratory.

3.3. Antibiotic Enhancing Effect. One of the exciting aspects of our studies is the observation that hMSCs may have the potential to serve as an adjunct therapeutic to conventional antibiotics. In addition to geneticin as a broad spectrum antibiotic, we investigated the ability of hMSCs supernatants to enhance the ability of ceftazadine and tobramycin on Pseudomonas aeruginosa growth (Figure 5, $100 \mu \mathrm{g} / \mathrm{mL}$ ). Ceftazadine and tobramycin are two antibiotics that are commonly used to treat Pseudomonas aeruginosa infections in patients with CF. Both ceftazadine and tobramycin were effective at decreasing Pseudomonas aeruginosa CFUs (Figure 5(a), $P \leq$ $0.05)$, as are the hMSC supernatants $(P \leq 0.07, n=4$ different hMSC donors). Combining respective antibiotic with hMSCs demonstrated an additive effect in decreasing Pseudomonas aeruginosa infection which was observed across a variety of antibiotic dosages (data not shown). Evaluating the different experiments for the overall impact on the relative \% change comparing antibiotic (either ceftazadine or tobramycin) with hMSC supernatant alone was statistically significant (Figure 5(b), $P \leq 0.05, n=4,100 \mu \mathrm{g} / \mathrm{mL}$ ).

3.4. Human Bone Marrow Derived MSCs versus Adipose Tissue Derived hMSCs. To determine if the antimicrobial effectiveness of the hMSCs is dependent on the tissue origin of the hMSCs, we investigated whether hMSCs derived from adipose tissue also demonstrated the potential for antibiotic enhancing activity. As with the bone marrow derived hMSCs, supernatants from adipose tissue derived hMSCs were also antimicrobial (Figure 6, $P<0.05, n=3$ different donors) decreasing Pseudomonas aeruginosa CFUs to levels comparable with the antibiotic control (geneticin). Further, when combining the geneticin with the adipose tissue derived hMSCs, the impact was consistently more potent against Pseudomonas aeruginosa than when using bone marrow derived hMSCs in combination with geneticin $(P \leq 0.05)$, which may be related to the donor or to the way in which each type of MSC was isolated and grown. Further, it should be noted that, in these subsets of experiments, the effectiveness of the MSCs augmented the antibiotic sensitivity; however, there was no demonstrated difference between the MSCs supernatant and the combined MSCs + geneticin. In addition, although the FD derived MSCs were antimicrobial and enhanced antibiotic effectiveness compared to geneticin alone, the significant variability minimized the difference of the combined therapeutics when compared to MSC supernatant alone. These observations emphasize the variability with different MSC preparations and the need for optimization.

3.5. I-172 versus Baseline Supernatants. Our previous data has suggested that bone marrow derived hMSCs deficient in 


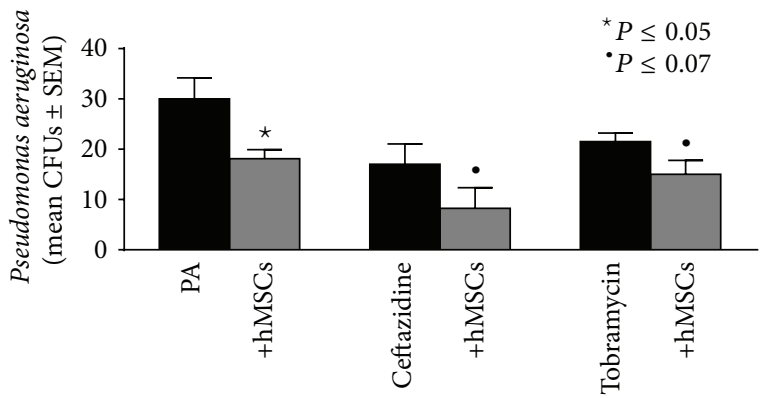

(a) hMSC supernatants enhance antibiotic effectiveness

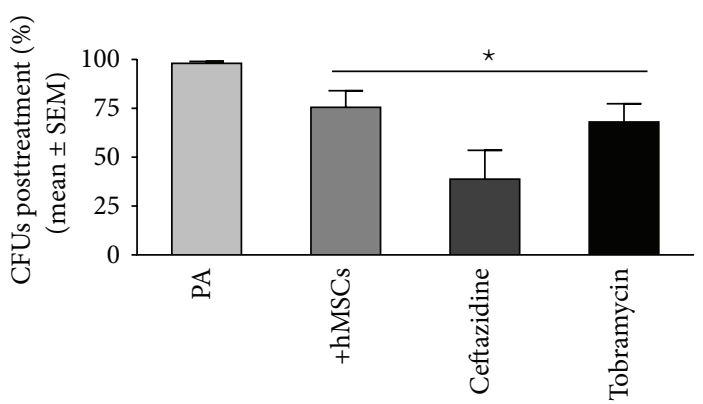

(b) Activity remaining posttreatment with hMSCs \pm antibiotics

FIGURE 5: Antibiotic enhancing activity. To determine whether the antibiotic enhancing effects of the bone marrow derived hMSCs were antibiotic specific, we tested the ability of the hMSCs to enhance the impact of other antibiotics which are often used to treat CF: ceftazadine $(100 \mu \mathrm{g} / \mathrm{mL})$ and tobramycin $(100 \mu \mathrm{g} / \mathrm{mL})$. (a) Ceftazadine and tobramycin decreased Pseudomonas aeruginosa CFUs $(P \leq 0.05, n=3)$ which were enhanced by the addition of hMSCs derived supernatants $(P=0.06$ for ceftazadine and $P=0.07$ for tobramycin). (b) When each experiment is used as its own control for Pseudomonas aeruginosa growth (100\%), the MSCs and the antibiotics had statistically significant effect on Pseudomonas growth $(100 \mu \mathrm{g} / \mathrm{mL}, P \leq 0.05)$.

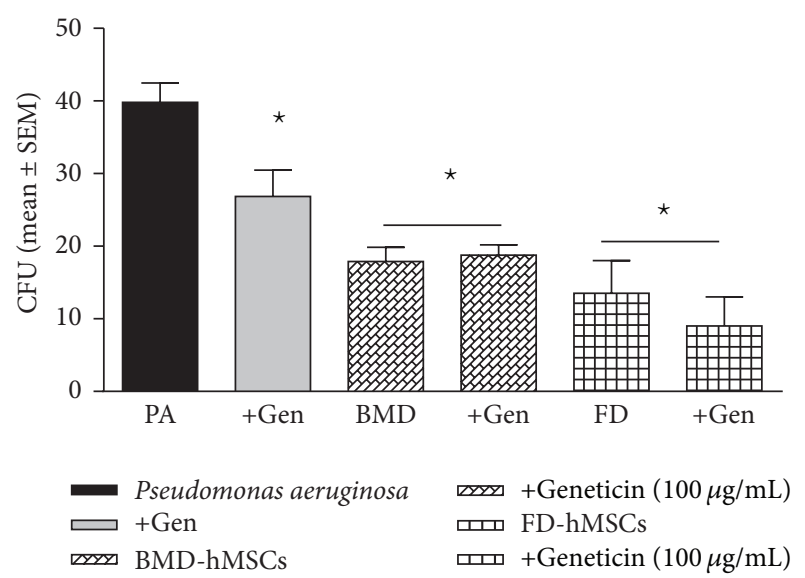

FIgURE 6: hMSC origin and impact on Pseudomonas aeruginosa growth. The availability of different hMSC sources provides the opportunity to explore whether the antimicrobial effectiveness of the hMSC supernatant was dependent on the tissue origin of the hMSC. Like the bone marrow derived hMSCs, hMSCs derived from adipose tissue significantly decreased Pseudomonas aeruginosa CFUs $(P \leq$ $0.05)$ and enhanced geneticin $(100 \mu \mathrm{g} / \mathrm{mL})$ effectiveness $(P=0.08$, $n=3$ ). $\mathrm{PA}=$ Pseudomonas aeruginosa growth without treatment. Geneticin = treatment with the antibiotic geneticin, +hMSCs = treatment with hMSC derived supernatant, and Both = treatment with both hMSC supernatants and geneticin. hMSC supernatants enhance antibiotic effectiveness against Pseudomonas aeruginosa.

CFTR function produce less LL-37 [8]. To determine whether hMSCs deficient in Cftr activity would have decreased antimicrobial activity, we tested supernatants from hMSCs that had been pretreated with the CFTR inhibitor I-172 (Sigma Chemical Co., $10 \mu \mathrm{g} / \mathrm{mL} ; 48$ hours). We confirmed that CFTR deficient hMSCs have less antimicrobial activity than normal hMSCs on Pseudomonas aeruginosa CFUs (Figure $7(\mathrm{a}), n=4, P \leq 0.05$ ) and alter growth rate (Figure $7(\mathrm{~b})$, $n=4, P \leq 0.05)$. In the Pseudomonas aeruginosa in vitro assays, CFUs were evaluated from I-172 treated and untreated
hMSC supernatants. hMSC supernatants decreased CFUs from $33 \pm 3.5$ to $28.5 \pm 8.5$ ( $P$ value) when compared to using hMSC supernatants generated in the presence of the CFTR inhibitor ( $38 \pm 16$ CFUs). Blocking hMSC CFTR activity also resulted in less efficient impact on decreasing the Pseudomonas aeruginosa growth rate. These data confirmed the inefficiency of CFTR deficient hMSC supernatants on decreasing Pseudomonas aeruginosa bacterial burden (Figure $7(\mathrm{~b}), P<0.05, n=4$ different hMSC donors) when compared to hMSC supernatants derived from hMSCs cultured without the CFTR inhibitor $(P<0.05, n=4$ different MSC donors). hMSC supernatants were also evaluated for the presence of the antimicrobial peptide LL-37 since the difference in antimicrobial effectiveness may be related to LL-37 activity. hMSCs which have CFTR blocked with the inhibitor I-172 secreted significantly less LL-37 compared to the hMSCs not treated with the CFTR inhibitor (Figure 7(c); $P \leq 0.05, n=5)$. This data implicates a relationship between LL-37 secretion, CFTR activity, antimicrobial effectiveness, and responsiveness of the hMSCs to bacterial pathogens.

3.6. Inducing $L L-37$. To determine if we could optimize hMSCs for antimicrobial potency, we evaluated the hMSCs for secretion and expression of the antimicrobial defensin LL37 , as well as human beta defensins (hBD) hBD-3 and hBD2 in response to a variety of effectors. None of the cultures, regardless of whether or not they were stimulated, contained (hBD-2 or hBD-3, (data not shown)) consistent with previous reports [9]. LL-37 is constitutively secreted by bone marrow derived hMSCs. The levels of LL-37 can be enhanced when the hMSCs are stimulated with effectors IFN $\gamma$, IL- $1 \beta$, or IL-12 (Figure $7(\mathrm{~d}), P \leq 0.05$ for each). Further, the source how the MSCs are grown for clinical use utilizing either serum-free conditions or platelet lysate conditions also had an impact on the in vivo antimicrobial efficacy of the hMSCs and production of LL-37. Unlike bone marrow derived hMSCs grown in fetal bovine serum, hMSCs grown under serumfree conditions or platelet lysate conditions produced no 


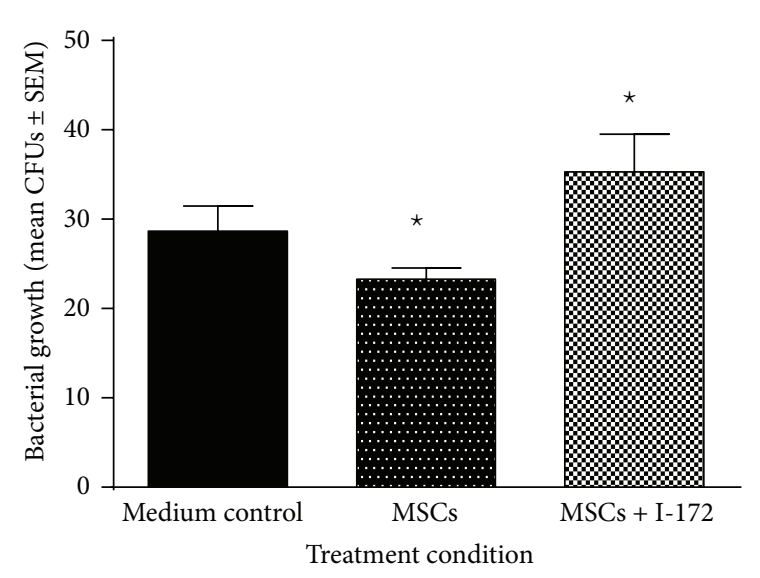

(a) Blocking CFTR function on Pseudomonas aeruginosa growth

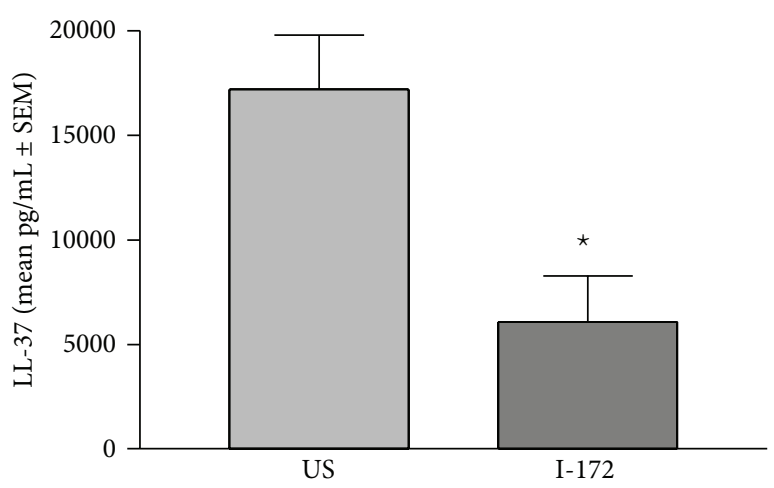

(c) Blocking CFTR function decreases LL-37 production

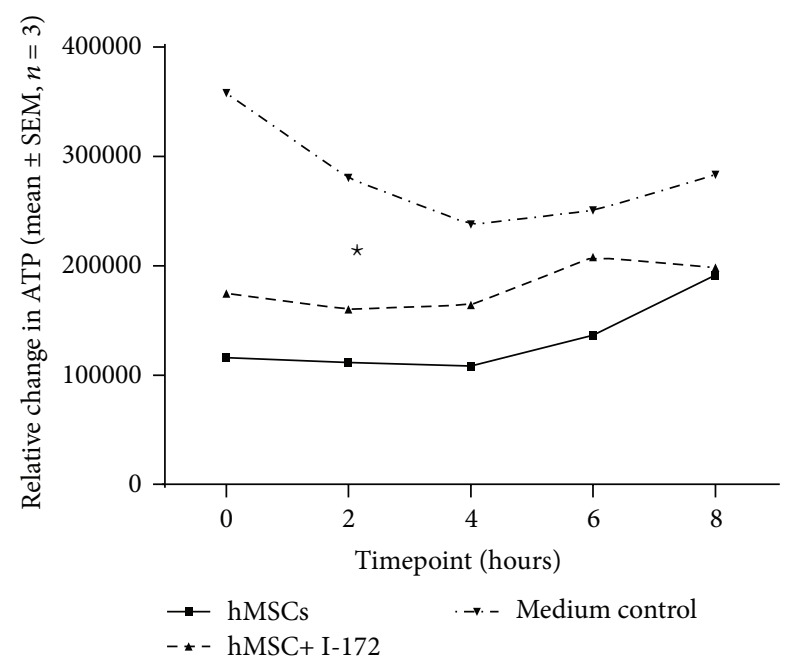

(b) Growth kinetics

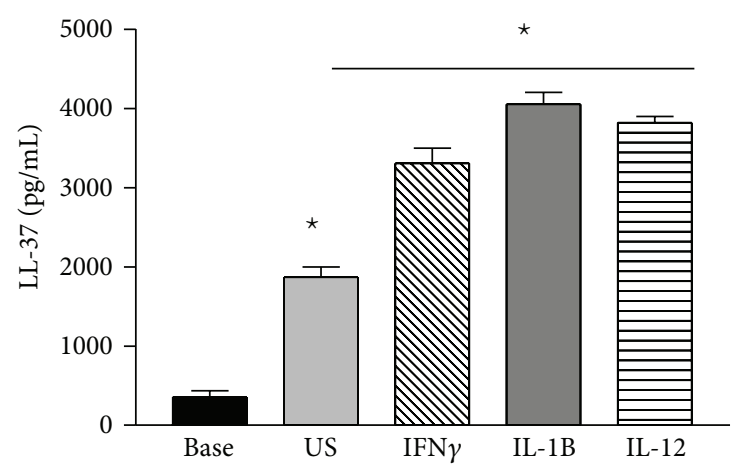

(d) hMSCs produce LL-37 in response to stimulation

FIGURE 7: Impact of blocking CFTR function on antimicrobial activity of MSCs. To mimic CF cells, healthy bone marrow derived hMSCs were cultured in the presence and absence of CFTR blocker I-172 $(10 \mu \mathrm{g} / \mathrm{mL})$ without antibiotics for 24 hours. The hMSC supernatants were evaluated for the ability to impact Pseudomonas aeruginosa PA CFUs (a) and growth rate (b). Supernatants generated from CFTR deficient hMSCs were more inefficient at decreasing Pseudomonas aeruginosa CFUs $((\mathrm{b}), P \leq 0.05)$ and growth rate $((\mathrm{c}), P \leq 0.05)$ than hMSCs without CFTR activity blocked. Further, hMSCs with deficient CFTR activity had less ability to secrete LL-37 ((c), $P \leq 0.05)$ relative to controls. LL-37 production by bone marrow derived hMSCs is decreased when CFTR is blocked but can be increased by treating the cells with a variety of cytokine stimulators. hMSCs stimulated with cytokines IFN $\gamma(100 \mathrm{ng} / \mathrm{mL})$, IL-1B (50 ng/mL), and IL-12 (100 ng/mL) secreted significantly more LL-37 than unstimulated controls ((d), $P \leq 0.05, n=4$ different hMSC preparations).

detectable LL-37 unless stimulated by LPS $(\geq 1000 \mathrm{pg} / \mathrm{mL}$, $P \leq 0.05)$ which was associated with the overall antimicrobial effectiveness. This may be why certain preparations of the MSCs have better antimicrobial and antibiotic enhancing activity than other preparations as observed in Figure 6; implicate the need for MSC optimization for clinical impact.

To begin to explore the mechanisms by which CFTR regulates LL-37 production, we took advantage of an immortalmouse derived MSC clone BMC9. These cells are abundant and have an MSC phenotype when grown at $37^{\circ} \mathrm{C}$. Further, we could actively quantify mouse Cftr using a highly reproducible mouse Cftr gene expression assay in our CF Animal CORE Center. The BMC9 cells have $36 \pm 14 \%$ Cftr expression (Ct value of $31 \pm 1$ ) compared to intestinal epithelium (Ct value equals $20 \pm 3$ ), with our sensitivity and specificity to detect Cftr at $40 \pm 2 \mathrm{Ct}$. BMC9 cells cultured with I-172 $(10 \mu \mathrm{g} / \mathrm{mL}$, for 48 hours $)$ expressed $37 \pm 13 \%$ less mouse
mCRAMP (mouse cathelicidin-LL-37 related antimicrobial peptide, mean \pm SEM, $n=3)$ than BMC9 cells not cultured with I-172 $(P \leq 0.05)$ [27]. These data suggest that hMSCs express CFTR and that CFTR function impacts the ability of hMSCs to produce LL-37. Studies are ongoing to establish how this might affect clinical impact.

\section{Discussion}

CF patients have increased susceptibility to pulmonary infections with Pseudomonas aeruginosa as well as pathogens such as Staphylococcus aureus and Streptococcus pneumoniae due to the unique environment created by deficient CFTR. The inability to resolve these infections and the ensuing severe inflammatory response is the major cause of morbidity and mortality in CF. Our previous data has shown that hMSCs decrease inflammation and infection in the in vivo murine 
model of CF chronic Pseudomonas aeruginosa infection and inflammation. In this paper we demonstrate that hMSCs have a beneficial impact on the Staphylococcus aureus in vivo murine model of CF lung infection and inflammation. In addition, we demonstrate that, in both in vivo and in vitro systems, hMSCs are beneficial in treating infections associated with both gram negative and gram positive pathogens and that the antimicrobial impact of the hMSCs can be associated with the antimicrobial peptide LL-37. The studies presented in this paper also focus on understanding the diversity in the hMSCs antimicrobial effectiveness and whether the hMSC effect is related to changing the overall growth rate of the bacteria depending on the type of pathogen. Further, we demonstrate the ability to optimize the hMSCs and their secreted products based upon source of tissue origin, since stimulation with a variety of stimulators or blocking CFTR activity alters the phenotype of the hMSCs.

Our studies demonstrate that (1) soluble products generated by hMSCs significantly decrease Pseudomonas aeruginosa, Staphylococcus aureus, and Streptococcus pneumoniae CFUs as well as having an impact on the growth rate of these pathogens; (2) the antimicrobial effectiveness of the hMSCs supernatants can enhance the effectiveness of antibiotics used to treat these types of infections; (3) hMSCs produce antimicrobial peptides such as LL-37 which are impacted by the function of CFTR or specific growth medium, which ultimately impacts the hMSC antimicrobial potency (measuring the strength of the hMSC effect) and efficacy (measuring the duration of the hMSC effect); (4) hMSCs derived from bone marrow or adipose tissue are both antimicrobial, suggesting that perhaps all hMSCs, regardless of their tissue origin, can be antimicrobial and could feasibly be optimized for therapeutic applications in infection.

The studies in this paper suggest that MSCs not only impact inflammation and infection but also are diverse at being able to slow the growth rate of a variety of bacteria. In vivo the hMSCs have a beneficial preclinical effect in both Pseudomonas aeruginosa and Staphylococcus aureus infection and inflammation murine models of CF. These studies imply that the hMSCs have the capacity to manage both gram negative and gram positive infections in CF, despite different levels of potency and efficacy. Further, the antimicrobial effectiveness is dependent on how the hMSCs are cultured which are likely different for each of the pathogens. Additionally, the antimicrobial and antibiotic enhancing activity of the MSCs appears to be dependent on how the MSCs are grown, donor variability, as well as the pathogen.

In terms of mechanisms related to the antimicrobial function of the hMSCs there are at least two ways in which the hMSC supernatants are impacting bacterial growth and activity. The studies in this paper suggest that the hMSCs are directly slowing down the growth activity of the bacteria resulting in a lower bacteria burden than what would otherwise result without the presence of the hMSC supernatants. The slowed growth and fewer bacteria can induce a window of opportunity for the antibiotics as well as the host's immune system to help resolve the infection. The concept of the hMSC supernatant impact on the bacteria can be broken down into at least two possible mechanisms. The first is by decreasing the overall bacterial burden or ability of the bacteria to overcome the antibiotic concentration. This allows for more efficient antibiotic effectiveness because the overall number of bacteria is more easily managed by the antibiotic treatment. The rate of the bacterial growth and the potency of the hMSC supernatant would be variables of the antimicrobial effect, which probably explains the differences in the overall kinetics of the hMSC supernatant impact on Pseudomonas aeruginosa, Staphylococcus aureus, and Streptococcus pneumoniae. This function of the hMSCs may be especially important in scenarios of sepsis, as well as CF, in which the bacteria burden, bacteria death by antibiotics, and release of proinflammatory stimulators contribute to the tissue damage $[28,29]$. In sepsis studies, the battle between prevention of bacterial overgrowth, the intensity of how the bacteria are killed, and the efficiency of the process is hypothesized to define the progression to death $[29,30]$.

The second piece of the important antimicrobial effectiveness of the hMSCs is the ability of the stem cells to secrete antimicrobial peptides including the peptide LL-37 $[8,9]$. Like other antimicrobial peptides, LL-37 mediates its effects by softening the bacterial cell wall and allowing increased sensitivity to host and antibacterial agents [31]. Since the impact of the LL-37 is on the bacterial wall, the potency of the hMSC antimicrobial effectiveness may be related to the type of pathogen $[32,33]$ which explains why the hMSC supernatants had different effects on Staphylococcus aureus and Streptococcus pneumoniae. For these two different pathogens it took longer to have an antimicrobial impact than when treating the Pseudomonas aeruginosa. This may be relative to the proportions and types of gram negative and gram positive organisms and the efficiency of both MSCs and LL-37 as well as a variety of other potential antimicrobial peptide products $[34,35]$. Further, the impact of the hMSC supernatants on improving geneticin sensitivity was not as potent, which could be related to the delayed impact of the hMSCs on the Staphylococcus aureus and Streptococcus pneumoniae growth kinetics. The action of the hMSCs and production of LL-37 may also be additive with the host response. hMSCs have the capacity to change the localize milieu eliciting a host response appropriate for the scenario at hand. Further, antimicrobial potency including additional sources of LL-37 may be induced in the host in response to the hMSCs. The hMSCs may induce an additive boost of antimicrobial efficiency harnessing both hMSCs and host effectiveness [31, 36, 37].

In this paper, we show that the production of LL-37 is related to the antimicrobial effectiveness of the hMSCs supernatants and that when the LL-37 is downregulated (when CFTR is blocked by the inhibitor I-172), the antimicrobial effectiveness of the supernatants becomes less efficient. There have been previous reports suggesting that LL-37 in scenarios of Pseudomonas aeruginosa infection or methicillin resistant Staphylococcus aureus and group B-Streptococcus pneumoniae (Streptococcus pyrogenes) may contribute to antibiotic sensitivity and regulate the production of quorum sensing molecules $[32,33]$ associated with the antimicrobial effectiveness of LL-37. Our studies were done with a clinical strain of mucoid Pseudomonas aeruginosa, non-MRSA 
Staphylococcus aureus, and Streptococcus pneumoniae with and without antibiotics which implies that close attention must be made in understanding hMSC and LL-37 therapy in scenarios of complex infections and source of pathogens. Further, additional properties of the hMSC supernatants may also be associated with antimicrobial potency and efficacy, including other antimicrobial molecules, although we did not detect the presence of defensins hBD-3 or hBD-2. This is the focus of on-going work in our laboratory. Our studies also suggest that recombinant LL-37 along with antibiotics may be a reasonable therapeutic for CF; however the high chloride environment of the CF lung does not sustain LL-37 functional activity $[35,38]$. Repeated dosages of recombinant LL-37 would have to be nebulized to keep active LL-37 in the lung. Based upon our studies, however, hMSCs may provide a unique therapeutic alternative as a continuous source of LL37 , to contribute to their environmental milieu [33, 34].

Another important observation from these studies is the similarities between the bone marrow derived hMSC supernatants and the adipose tissue derived hMSC supernatants in antimicrobial activity. The tissue origin of the hMSCs is likely to reflect where the MSCs reside, their initial niche of differentiation and surrounding microenvironment $[39,40]$. Our data shows that although bone marrow derived and adipose derived hMSCs come from different environmental niches, both sources of hMSCs possess important antimicrobial properties. These studies support that hMSCs, whether they are derived from bone marrow or adipose tissue, have the potential to have imminent therapeutic impact in scenarios of infection and inflammation.

Our data also suggests that, in terms of hMSC therapy in CF, the patient's own cells may not be as efficient at antimicrobial activity as MSC derived from healthy individuals. hMSCs with deficient CFTR function produce less LL-37, which has the capacity to contribute to inefficient Pseudomonas aeruginosa killing. This suggests that the patients' own cells may not be as efficient in the host response to infection. Further, whether deficient hMSC LL-37 is part of the pathophysiology related to the inability of the CF patients to resolve infection and whether supplemental therapeutics with hMSCs from healthy donors may be beneficial remain to be determined. Our laboratories are actively studying this observation since it has implications not only for CF but also for other diseases. These data also suggest that environment and phenotype are important factors in determining the overall beneficial effect of hMSCs in resolving bacterial infections. The optimal conditions for generating an hMSC phenotype with the most efficient antimicrobial and antibiotic enhancing effectiveness have yet to be identified and are the focus of high-throughput studies to identify the hMSC response versus function for clinical applications.

From these studies, we can conclude that (1) hMSC therapy improves outcomes in CF lung infection with both Pseudomonas aeruginosa and Staphylococcus aureus in vivo determined by the murine model of CF lung infection and inflammation; (2) hMSC supernatants significantly decreased the growth of Pseudomonas aeruginosa, Staphylococcus aureus, and Streptococcal pneumoniae in vitro; (3) both sources of MSCs have the capacity to have antimicrobial and antibiotic enhancing activity which is dependent on the donor as well as growth conditions in the preparations of the hMSC; (4) hMSCs secreted bioactive molecules, including LL-37, but not hBD-3 or hBD-2, which is consistent with antimicrobial effectiveness; (5) the impact of hMSCs on antibiotics appears to be concentration and time course dependent, as well as MSC source dependent and pathogen specific; (6) growth conditions significantly impact the overall antimicrobial impact such as altering MSC-CFTR activity or stimulating with a variety of cytokines when compared to MSCs not treated with any stimulant; (7) hMSCs with deficient CFTR activity are not as efficient at handling infection as hMSCs with sufficient CFTR activity when evaluated using in vitro antimicrobial assays monitoring pathogen growth kinetics and CFUs.

\section{Summary}

The studies outlined in this paper demonstrate the diverse antimicrobial and antibiotic enhancing potency of hMSCs and their products. MSCs as therapeutic powerhouses may require optimization for the greatest clinical impact. Further, the studies in this paper emphasize the significant clinical potential of MSCs for treating infections like those associated with cystic fibrosis chronic lung disease and support our Phase I Clinical Trial investigating the safety of hMSCs in patients with CF.

\section{Conflict of Interests}

The authors have no conflict of interests to report.

\section{Acknowledgments}

The authors appreciate the expertise and the services provided by the CTSC Bioanalyte Core and the CF Animal CORE. The funding for this research was graciously provided by the David and Virginia Baldwin Fund, The National Center for Stem Cell and Regenerative Medicine, The Case Western Reserve Vision Fund, National Institutes of Health HL: R2104362, and the Cystic Fibrosis Foundation: BONFIE1410.

\section{References}

[1] J. F. Chmiel and M. W. Konstan, "Anti-inflammatory medications for cystic fibrosis lung disease: selecting the most appropriate agent," Treatments in Respiratory Medicine, vol. 4, no. 4, pp. 255-273, 2005.

[2] D. P. Nichols, M. W. Konstan, and J. F. Chmiel, "Antiinflammatory therapies for cystic fibrosis-related lung disease," Clinical Reviews in Allergy and Immunology, vol. 35, no. 3, pp. 135-153, 2008.

[3] J. F. Chmiel and M. W. Konstan, "Inflammation and anti-inflammatory therapies for cystic fibrosis," Clinics in Chest Medicine, vol. 28, no. 2, pp. 331-346, 2007.

[4] D. J. Weiss, M. A. Berberich, Z. Borok et al., "Adult stem cells, lung biology, and lung disease. NHLBI/Cystic Fibrosis Foundation Workshop," Proceedings of the American Thoracic Society, vol. 3, no. 3, pp. 193-207, 2006. 
[5] A. I. Caplan, "Why are MSCs therapeutic? New data: new insight," Journal of Pathology, vol. 217, no. 2, pp. 318-324, 2009.

[6] B. M. Abdallah and M. Kassem, "Human mesenchymal stem cells: from basic biology to clinical applications," Gene Therapy, vol. 15, no. 2, pp. 109-116, 2008.

[7] A. I. Caplan and J. E. Dennis, "Mesenchymal stem cells as trophic mediators," Journal of Cellular Biochemistry, vol. 98, no. 5, pp. 1076-1084, 2006.

[8] T. L. Bonfield, D. Lennon, S. K. Ghosh, A. M. DiMarino, A. Weinberg, and A. I. Caplan, "Cell based therapy aides in infection and inflammation resolution in the murine model of cystic fibrosis lung disease," Stem Cell Discovery, vol. 3, no. 2, pp. 139-153, 2013.

[9] A. Krasnodembskaya, Y. Song, X. Fang et al., "Antibacterial effect of human mesenchymal stem cells is mediated in part from secretion of the antimicrobial peptide LL-37,' Stem Cells, vol. 28, no. 12, pp. 2229-2238, 2010.

[10] A. Bragonzi, I. Farulla, M. Paroni et al., "Modelling co-infection of the cystic fibrosis lung by Pseudomonas aeruginosa and Burkholderia cenocepacia reveals influences on biofilm formation and host response," PLoS ONE, vol. 7, no. 12, Article ID e52330, 2012.

[11] S. D. Sagel, J. F. Chmiel, and M. W. Konstan, "Sputum biomarkers of inflammation in cystic fibrosis lung disease," Proceedings of the American Thoracic Society, vol. 4, no. 4, pp. 406-417, 2007.

[12] T. L. Bonfield, M. T. Nolan, D. P. Lennon, and A. I. Caplan, "Defining human mesenchymal stem cell efficacy in vivo," Journal of Inflammation, vol. 7, article 51, 2010.

[13] D. P. Lennon and A. I. Caplan, "Isolation of human marrowderived mesenchymal stem cells," Experimental Hematology, vol. 34, no. 11, pp. 1604-1605, 2006.

[14] Y.-T. Chen, C.-K. Sun, Y.-C. Lin et al., "Adipose-derived mesenchymal stem cell protects kidneys against ischemiareperfusion injury through suppressing oxidative stress and inflammatory reaction," Journal of Translational Medicine, vol. 9, article 51, 2011.

[15] A. M. LeVine, K. E. Kurak, J. R. Wright et al., "Surfactant protein-a binds group B streptococcus enhancing phagocytosis and clearance from lungs of surfactant protein-a-deficient mice," American Journal of Respiratory Cell and Molecular Biology, vol. 20, no. 2, pp. 279-286, 1999.

[16] A. Heeckeren, R. Walenga, M. W. Konstan, T. Bonfield, P. B. Davis, and T. Ferkol, "Excessive inflammatory response of cystic fibrosis mice to bronchopulmonary infection with Pseudomonas aeruginosa," Journal of Clinical Investigation, vol. 100, no. 11, pp. 2810-2815, 1997.

[17] H. A. Cash, D. E. Woods, B. McCullough, W. G. Johanson Jr., and J. A. Bass, "A rat model of chronic respiratory infection with Pseudomonas aeruginosa," American Review of Respiratory Disease, vol. 119, no. 3, pp. 453-459, 1979.

[18] N. K. Archer, M. J. Mazaitis, J. W. Costerton, J. G. Leid, M. E. Powers, and M. E. Shirtliff, "Staphylococcus aureus biofilms: properties, regulation, and roles in human disease," Virulence, vol. 2, no. 5, pp. 445-459, 2011.

[19] S. K. Ghosh, T. A. Gerken, K. M. Schneider, Z. Feng, T. S. McCormick, and A. Weinberg, "Quantification of human $\beta$ defensin-2 and -3 in body fluids: application for studies of innate immunity," Clinical Chemistry, vol. 53, no. 4, pp. 757-765, 2007.

[20] H. I. Kawsar, S. K. Ghosh, S. A. Hirsch, H. B. Koon, A. Weinberg, and G. Jin, "Expression of human beta-defensin-2 in intratumoral vascular endothelium and in endothelial cells induced by transforming growth factor beta," Peptides, vol. 31, no. 2, pp. 195-201, 2010.

[21] R. Simon, M. D. Radmacher, and K. Dobbin, "Design of studies using DNA microarrays," Genetic Epidemiology, vol. 23, no. 1, pp. 21-36, 2002.

[22] B. A. Simon, R. B. Easley, D. N. Grigoryev et al., "Microarray analysis of regional cellular responses to local mechanical stress in acute lung injury," American Journal of Physiology: Lung Cellular and Molecular Physiology, vol. 291, no. 5, pp. L851-L861, 2006.

[23] M. L. Drumm, M. W. Konstan, M. D. Schluchter et al., "Genetic modifiers of lung disease in cystic fibrosis," The New England Journal of Medicine, vol. 353, no. 14, pp. 1443-1453, 2005.

[24] M. D. Schluchter, M. W. Konstan, M. L. Drumm, J. R. Yankaskas, and M. R. Knowles, "Classifying severity of cystic fibrosis lung disease using longitudinal pulmonary function data," American Journal of Respiratory and Critical Care Medicine, vol. 174, no. 7, pp. 780-786, 2006.

[25] A. D. Guerra, D. A. Cantu, J. T. Vecchi, W. E. Rose, P. Hematti, and W. J. Kao, "Mesenchymal stromal/stem cell and minocycline-loaded hydrogels inhibit the growth of Staphylococcus aureus that evades immunomodulation of blood-derived leukocytes," The AAPS Journal, vol. 17, no. 3, pp. 620-630, 2015.

[26] M. A. Matthay, A. Goolaerts, J. P. Howard, and J. W. Lee, "Mesenchymal stem cells for acute lung injury: preclinical evidence," Critical Care Medicine, vol. 38, no. 10, pp. S569-S573, 2010.

[27] M. A. Kovach, M. N. Ballinger, M. W. Newstead et al., "Cathelicidin-related antimicrobial peptide is required for effective lung mucosal immunity in gram-negative bacterial pneumonia," Journal of Immunology, vol. 189, no. 1, pp. 304-311, 2012.

[28] A. Lever and I. Mackenzie, "Sepsis: definition, epidemiology, and diagnosis," British Medical Journal, vol. 335, no. 7625, pp. 879-883, 2007.

[29] L. M. Napolitano, "Immune stimulation in sepsis: to be or not to be?” Chest, vol. 127, no. 6, pp. 1882-1885, 2005.

[30] K. Lyn-Kew and T. J. Standiford, "Immunosuppression in sepsis," Current Pharmaceutical Design, vol. 14, no. 19, pp. 1870$1881,2008$.

[31] M. Golec, "Cathelicidin LL-37: LPS-neutralizing, pleiotropic peptide," Annals of Agricultural and Environmental Medicine, vol. 14, no. 1, pp. 1-4, 2007.

[32] G. Sakoulas, K. Guram, K. Reyes, V. Nizet, and M. Zervos, "Human cathelicidin LL-37 resistance and increased daptomycin MIC in methicillin-resistant Staphylococcus aureus strain USA600 (ST45) are associated with increased mortality in a hospital setting," Journal of Clinical Microbiology, vol. 52, no. 6, pp. 2172-2174, 2014.

[33] N. Strempe, A. Neidig, M. Nusser et al., "Human host defense peptide LL-37 stimulates virulence factor production and adaptive resistance in Pseudomonas aeruginosa," PLoS ONE, vol. 8, no. 12, Article ID e82240, 2013.

[34] A. Scott, S. Weldon, P. J. Buchanan et al., "Evaluation of the ability of LL-37 to neutralise LPS in vitro and ex vivo," PLoS ONE, vol. 6, no. 10, Article ID e26525, 2011.

[35] K. L. Brown, G. F. T. Poon, D. Birkenhead et al., "Host defense peptide LL-37 selectively reduces proinflammatory macrophage responses," Journal of Immunology, vol. 186, no. 9, pp. 54975505, 2011. 
[36] P. Méndez-Samperio, "The human cathelicidin hCAP18/LL-37: a multifunctional peptide involved in mycobacterial infections," Peptides, vol. 31, no. 9, pp. 1791-1798, 2010.

[37] J. J. Auletta, S. K. Eid, P. Wuttisarnwattana et al., "Human mesenchymal stromal cells attenuate graft-versus-host disease and maintain graft-versus-leukemia activity following experimental allogeneic bone marrow transplantation," STEM CELLS, vol. 33, no. 2, pp. 601-614, 2014.

[38] C. I.-U. Chen, S. Schaller-Bals, K. P. Paul, U. Wahn, and R. Bals, " $\beta$-defensins and LL-37 in bronchoalveolar lavage fluid of patients with cystic fibrosis," Journal of Cystic Fibrosis, vol. 3, no. 1, pp. 45-50, 2004.

[39] D. S. Zagoura, O. Trohatou, V. Bitsika et al., "AF-MSCs fate can be regulated by culture conditions," Cell Death and Disease, vol. 4, article e571, 2013.

[40] E. Soleymaninejadian, K. Pramanik, and E. Samadian, "Immunomodulatory properties of mesenchymal stem cells: cytokines and factors," American Journal of Reproductive Immunology, vol. 67, no. 1, pp. 1-8, 2012. 

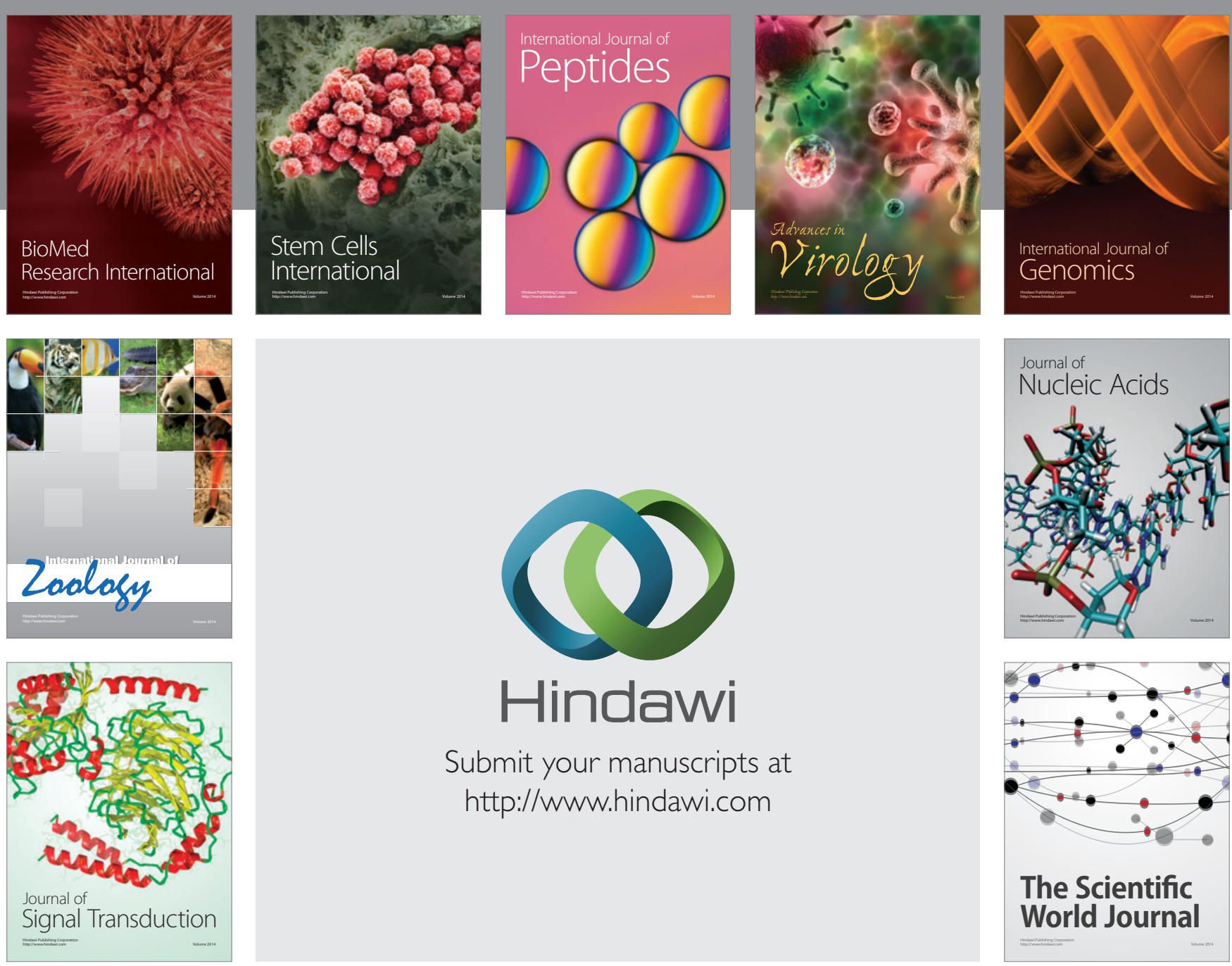

Submit your manuscripts at

http://www.hindawi.com
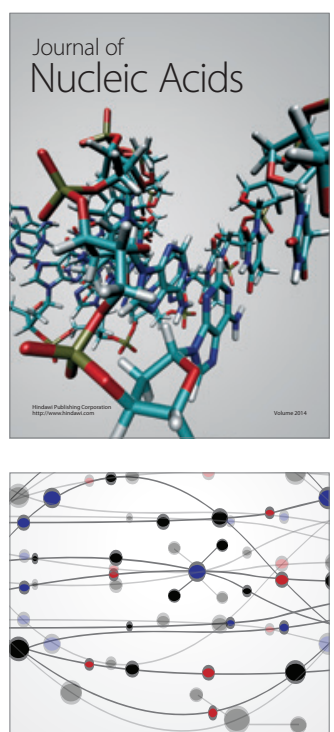

The Scientific World Journal
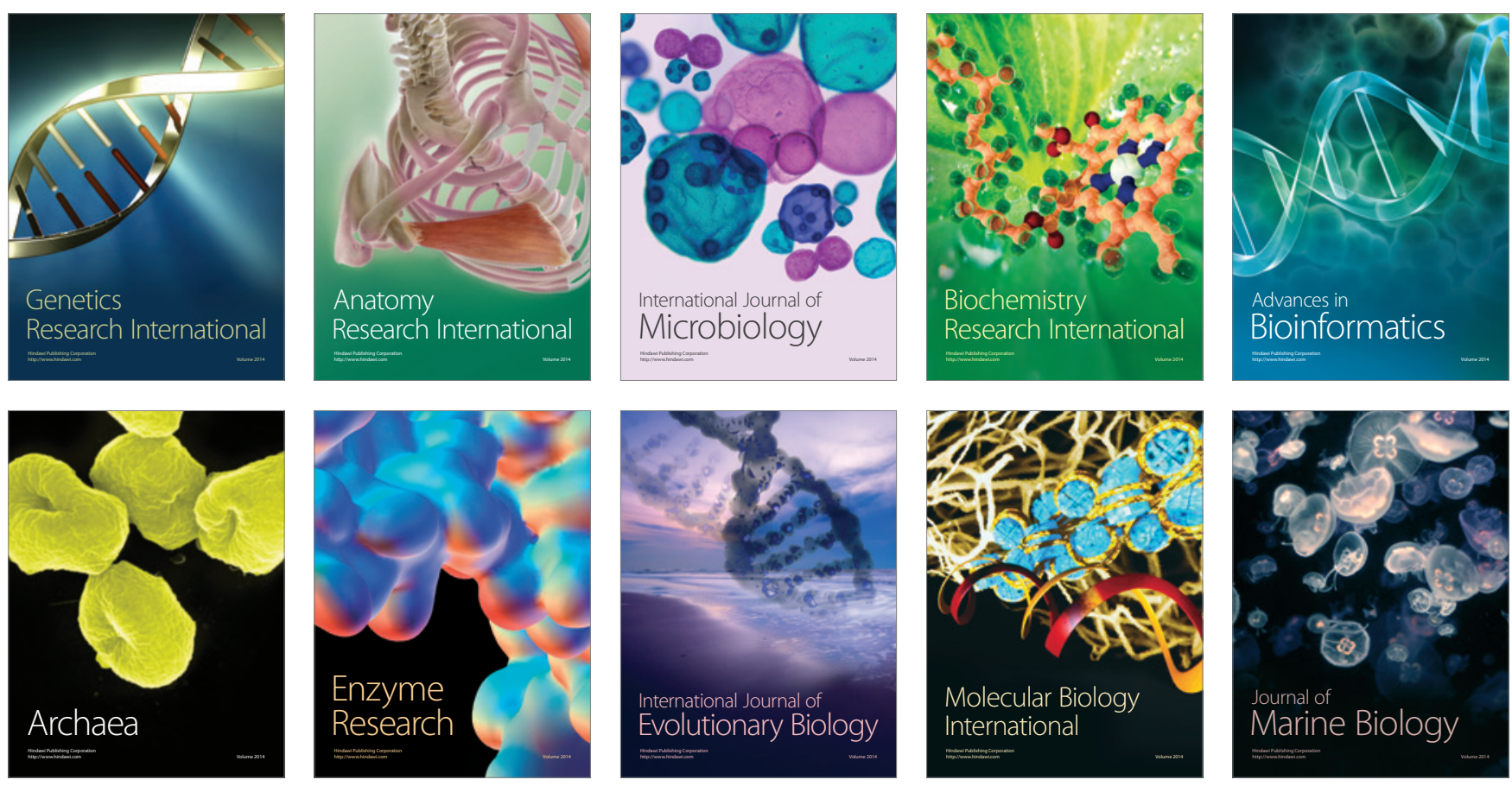